\title{
Article \\ Platelet Activation and Inflammation in Patients with Papillary Thyroid Cancer
}

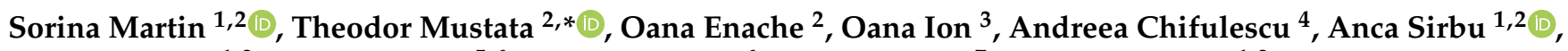 \\ Carmen Barbu ${ }^{1,2}$, Adrian Miron ${ }^{5,6}$, Cosmin Giulea ${ }^{6}$, Florin Andrei ${ }^{7}$ and Simona Fica ${ }^{1,2}$
}

1 Endocrinology Department, Carol Davila University of Medicine and Pharmacy, 020021 Bucharest, Romania; sorina.martin@gmail.com (S.M.); ancaelenasirbu@yahoo.com (A.S.); carmen_gabriela_barbu@yahoo.co.uk (C.B.); simonafica55@gmail.com (S.F.)

2 Endocrinology Department, Elias Hospital, 011461 Bucharest, Romania; oana.budianu@yahoo.ro

3 Nephrology Department, Fundeni Clinical Institute, 022328 Bucharest, Romania; oana.catalina_ion@yahoo.ro

4 Gastroenterology Department, Fundeni Clinical Institute, 022328 Bucharest, Romania; andreea.grigore92@yahoo.com

5 Surgery Department, Carol Davila University of Medicine and Pharmacy, 020021 Bucharest, Romania; dramiron@yahoo.com

6 Surgery Department, Elias Hospital, 011461 Bucharest, Romania; gcosmin@yahoo.com

7 Pathology Department, Elias Hospital, 011461 Bucharest, Romania; florina6@yahoo.com

* Correspondence: theo.mustata@gmail.com

check for

updates

Citation: Martin, S.; Mustata, T.; Enache, O.; Ion, O.; Chifulescu, A.; Sirbu, A.; Barbu, C.; Miron, A.;

Giulea, C.; Andrei, F.; et al. Platelet Activation and Inflammation in Patients with Papillary Thyroid Cancer. Diagnostics 2021, 11, 1959. https: / / doi.org/10.3390/

diagnostics11111959

Academic Editor: Luca Giovanella

Received: 2 October 2021

Accepted: 19 October 2021

Published: 22 October 2021

Publisher's Note: MDPI stays neutral with regard to jurisdictional claims in published maps and institutional affiliations.

Copyright: (c) 2021 by the authors. Licensee MDPI, Basel, Switzerland. This article is an open access article distributed under the terms and conditions of the Creative Commons Attribution (CC BY) license (https:/ / creativecommons.org/licenses/by/ $4.0 /)$.

\begin{abstract}
Background: The primary endpoint was to analyze the preoperatory inflammatory markers and platelet indices in papillary thyroid cancer (PTC) patients compared with patients with benign thyroid pathology. The secondary endpoints were to analyze the relationship between these markers and the pathological features of PTC and to compare their pre- and postoperative levels in PTC patients. Methods: In this retrospective case-control study, we analyzed the files of 1183 patients submitted to thyroidectomy between January 2012 and December 2018. A total of 234 patients with PTC (mean age $51.54 \pm 13.10$ years, $84.6 \%$ females) were compared with an age-, genderand BMI-matched control group of 108 patients with histologic benign thyroid disorders. Results: PTC patients had higher platelet count (PLT) $(p=0.011)$, plateletcrit (PCT) $(p=0.006)$, neutrophil $(p=0.022)$ and fibrinogen $(p=0.005)$ levels. Subgroup analysis showed that PTC females had higher PLT $(p=0.006)$, PCT $(p<0.001)$ and erythrocyte sedimentation rate (ESR) $(p=0.005)$, while males had higher neutrophil $(p=0.040)$ levels. Papillary thyroid cancer patients under 55 years had higher PLT $(p<0.001)$ and PCT $(p=0.010)$, while patients over 55 years had higher mean platelet volume $(p=0.032)$, neutrophil-to-lymphocyte ratio $(p=0.013)$, ESR $(p=0.005)$ and fibrinogen $(p=0.019)$ levels. Preoperative values for platelet indices and inflammatory markers were similar to the postoperative determinations in PTC patients. Fibrinogen (AUROC $=0.602, p=0.02$; cut-off $=327.5 \mathrm{mg} / \mathrm{dL}$, Se $=53.8 \%, \mathrm{Sp}=62.9 \%)$ and PLT $\left(\right.$ AUROC $=0.584, p=0.012 ;$ cut-off $=223.5 \times 10^{3} / \mathrm{mm}^{3}$, Se $=73.1 \%$, $\mathrm{Sp}=42.6 \%$ ) were independent predictors of the presence of PTC. Conclusions: Our data show that fibrinogen and platelet count could be promising, inexpensive, independent predictors for the presence of PTC when compared with benign thyroid disorders.
\end{abstract}

Keywords: differentiated thyroid cancer; papillary thyroid cancer; inflammatory markers; platelet indices

\section{Introduction}

Thyroid carcinomas, the most common type of endocrine malignancies, are 2-4 times more common in women than men, and their incidence has rapidly increased during the past decades, mostly due to increased incidence of papillary thyroid carcinomas (PTCs) [1]. The prevalence of thyroid nodules ranges from $5 \%$ by palpation to $19-68 \%$ by high resolution ultrasonography [2]. Ultrasonography and fine-needle aspiration biopsy play important roles in the diagnosis and follow-up of thyroid nodules. In addition, a variety 
of other parameters have been investigated in order to differentiate between benign and malignant thyroid nodules and to select appropriate treatments.

Although the relationship between cancer and inflammation has been known since 1863 when Rudolf Virchow hypothesized that chronic inflammation could contribute to the tumorigenic process, increasing attention has been recently focused on inflammation and immunity as major mechanisms involved in thyroid tumorigenesis [3]. Furthermore, chronic inflammation plays important roles both in tumor progression as well as in immune surveillance and responses to therapy. The possible mechanisms by which inflammation can contribute to carcinogenesis include induction of genomic instability, alterations in epigenetic events and subsequent inappropriate gene expression, enhanced proliferation of initiated cells, resistance to apoptosis, aggressive tumor neovascularization, invasion through tumor-associated basement membrane and metastasis [4-6]. Conversely, the tumor itself promotes an inflammatory reaction by secretion of proinflammatory factors or by development of tumor necrosis $[7,8]$.

The relationship between platelets (PLT) and cancer is not fully understood. Nonetheless, recent studies show that platelets and malignant cells influence one another. Malignant cells stimulate platelets production (paraneoplastic thrombocytosis) and cause platelets activation and aggregation. Conversely, platelets sustain tumor growth, tissue invasion and metastases [9].

Neutrophil count, lymphocyte count, the neutrophil-to-lymphocyte ratio (NLR), the lymphocyte-to-monocyte ratio (LMR), PLTs and platelet indices (platelet distribution width (PDW), plateletcrit (PCT) and mean platelet volume (MPV)), as indicators of platelet morphology and activation, could be used as inflammatory markers in cancer patients in addition to their use in cardiovascular, cerebrovascular, thromboembolic and inflammatory diseases [10]. Nevertheless, these potentially related carcinoma markers are inconsistent in PTC patients, with studies reporting discrepant results. Seretis et al. were one of the first groups to study the potential association of PTC and NLR and supposed that NLR may predict the presence of occult papillary thyroid microcarcinoma (PTMC) in otherwise benign goiters [11]. Gong et al. also reported that the preoperative NLR was closely related to the stage of papillary thyroid carcinoma [12]. NLR was also shown to be a prognostic factor for recurrence in low-risk DTC patients [13], and a correlation between NLR and tumor size in thyroid cancers has been shown by Cheong et al. [14]. Additionally, recent studies suggest that platelet indices may be possible markers in the differential diagnosis of benign and malignant thyroid disorders. MPV values were found to be significantly higher in patients with PTC compared with healthy patients or patients with nodular goiter [15]. There was also reported a significant decrease in MPV after thyroidectomy in patients with PTC, which was not observed in patients with nodular goiter [16]. Lower PDW and higher PCT values were observed in PTC patients when compared with healthy and benign thyroid disordered patients [17]. Li et al. recently suggested that the combined use of PDW and albumin might be useful in distinguishing thyroid cancer from benign thyroid nodules [18]. More than that, Wen et al. reported that pretreatment peripheral indexes, such as neutrophils, lymphocytes, MPV, PDW and LMR, could function as inexpensive indicators of aggressive behavior and higher stage in elderly patients with PTC [19]. However, there are studies that found no significant relationship between PTC and inflammatory hematological parameters including, in particular, NLR and MPV [20]. Machairas et al. found that white blood cell and platelet indices cannot assist in distinguishing benign goiter from thyroid cancer, but, in PTC patients, they can provide information about tumor multifocality and extrathyroidal extension [21]. Manatakis et al. also stated that NLR and platelet-to-lymphocyte ratios (PLR) cannot effectively predict the presence of occult papillary microcarcinomas in benign, multinodular goiters [22].

Therefore, the primary endpoint of the present retrospective study was to analyze the preoperatory inflammatory markers and platelet indices in PTC patients compared with patients with benign thyroid pathology. The secondary endpoints were to analyze the relationship between inflammatory markers and platelet indices and pathological features 
of PTC and to compare pre- and postoperative inflammatory markers and platelet indices in PTC patients.

\section{Materials and Methods}

\subsection{Patients and Study Protocol}

We conducted a hospital-based retrospective case-control study using the data from 1183 files of patients who underwent total thyroidectomy or lobectomy in our surgery department between January 2012 and December 2018. A total of $265(22.40 \%)$ of these patients were diagnosed with thyroid cancer. The study population included $249(93.96 \%)$ patients with histologic differentiated thyroid cancer (DTC), 234 (88.3\%) papillary thyroid carcinomas and 15 (5.66\%) follicular thyroid carcinomas (FTC). Exclusion criteria were poorly differentiated thyroid cancers $(4,1.51 \%)$, anaplastic cancers $(4,1.51 \%)$, medullary thyroid carcinomas (MTC) $(8,3.02 \%)$ and patients with histologic benign thyroid pathology (918). The controls were selected from the same cohort of 1183 patients and included the first 108 age-, gender- and BMI-matched subjects with histologic benign thyroid pathology: multinodular goiter (84), follicular adenoma (12) and Graves' disease (12) (Figure 1). Due to the retrospective nature of the study and the recent change in nomenclature that saw EFVPTC (encapsulated follicular variant of papillary thyroid carcinoma) redefined as NIFTP (noninvasive follicular thyroid neoplasm with papillary-like nuclear features) [23], we did not include patients diagnosed with NIFTP after 2016 and did not analyze patients based on the PTC histological subtype.

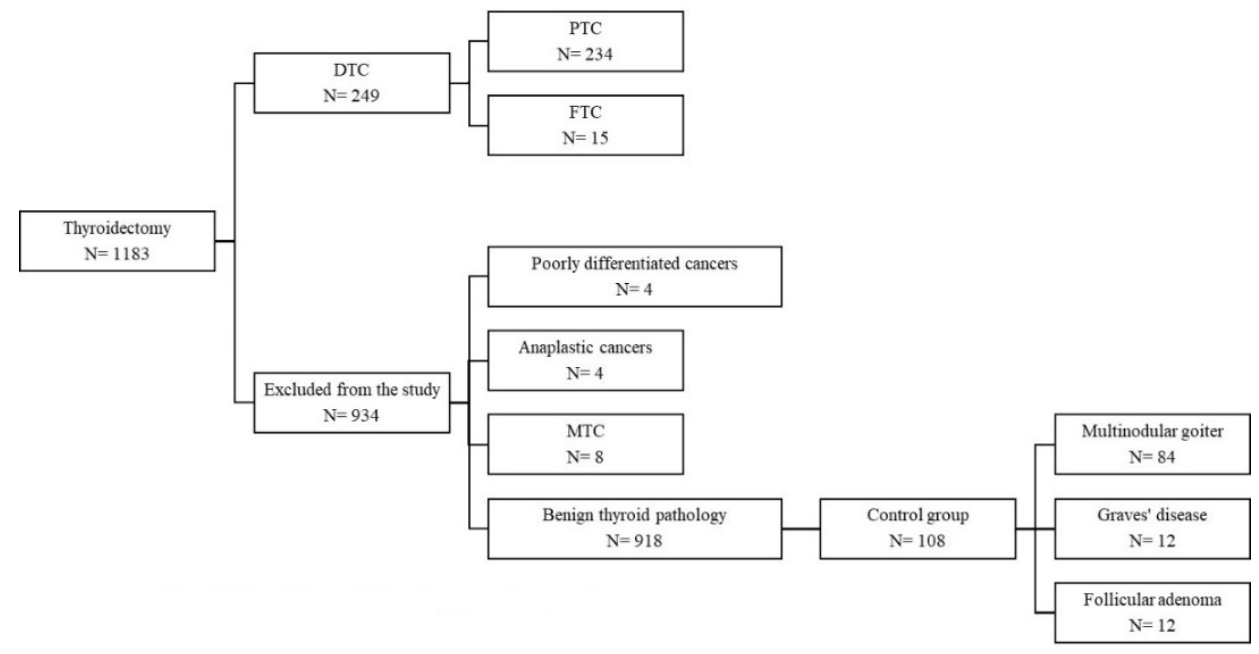

Figure 1. Flow diagram of the patient selection procedure.

For all the 249 DTC patients, we recorded the following preoperative clinical and paraclinical data: age, gender, body mass index (BMI), fibrinogen, erythrocyte sedimentation rate (ESR), platelet count, MPV, PDW, PCT, TSH, FT4, anti- thyroid peroxidase antibodies (TPOAb), anti- thyroglobulin antibodies (ATA), anti-TSH receptor antibodies (TRAb) and pathological report data describing tumor features that included pathological variant of DTC-follicular and papillary carcinoma subtypes, cancer size, pTNM stage [21], multifocality, vascular invasion, capsular invasion, extracapsular extension, locoregional lymph node involvement and presence of thyroiditis. In addition, we calculated the neutrophil-tolymphocyte (NLR) and platelet-to-lymphocyte (PLR) ratios as follows: NLR was calculated as the absolute neutrophil count divided by the absolute lymphocyte count, and PLR was calculated as the absolute platelet count divided by the absolute lymphocyte count, based on the preoperative blood count. For 63 PTC patients, the following postoperative data were also available to be recorded at a mean time of 3 months after surgery at the first follow-up visit: ESR, platelet count, MPV, PDW, PCT. For the 108 patients with benign thyroid pathology who were included in the control group, preoperative data were col- 
lected: age, gender, BMI, fibrinogen, ESR, platelet count, MPV, PDW, PCT, NLR, PLR and pathological report diagnosis.

Fasting baseline blood samples were routinely obtained the day before surgery and included hematocrit, hemoglobin, total WBC, automated differential counts (neutrophils, lymphocytes, monocytes, basophils and eosinophils) and platelets using a Sysmex XN-1000TM Hematology Analyzer (Sysmex Corporation, Kobe, Japan). Normal values for platelet indices were as follows: platelet count $=150-400 \times 10^{3} / \mathrm{mm}^{3} ; \mathrm{MPV}=6.7-11.5 \mathrm{fL} ; \mathrm{PDW}=10.1-16.1 \%$; $\mathrm{PCT}=0.17-0.32 \%$. Normal ESR $=0-20 \mathrm{~mm} / \mathrm{h}$ and fibrinogen $=238-498 \mathrm{mg} / \mathrm{dL}$. Serum TSH, FT4 and anti-thyroid antibodies were evaluated in a single laboratory by autoimmune amplified chemiluminescence immunochemistry using Immulite 2000 (Siemens Healthcare Diagnostics Products Ltd., Erlangen, Germany). Antithyroid antibody status was defined as follows: ATA positive $=$ patients with ATA serum levels $>40 \mathrm{IU} / \mathrm{mL}$; TPOAb positive $=$ patients with TPOAb serum levels $>35 \mathrm{IU} / \mathrm{mL} ; \mathrm{TRAb}$ positive $=$ patients with TRAb serum levels $>1.5 \mathrm{IU} / \mathrm{L}$. Autoimmune thyroiditis was diagnosed based on thyroid antibody positivity and/or the pathological diagnosis.

\subsection{Data Presentation and Statistical Analyses}

The distribution of the continuous quantitative variables was evaluated by the ShapiroWilk normality test. Descriptive data are presented as means \pm SD, medians with interquartile range (IQR) or percentage. Between groups comparisons were carried out using parametric (independent sample $t$-test, $t$-test for pairwise samples, one-way analysis of variance (ANOVA) for more than two independent groups) or nonparametric (Mann-Whitney U-test, Kruskal-Wallis one-way ANOVA, Kolmogorov-Smirnov) tests, as appropriate. Chisquare test and Fisher's exact test were used to compare proportions in large, respectively small groups. Relations between continuous variables were analyzed using Pearson's correlation parametric coefficient or Spearman's rho nonparametric correlation coefficient. Linear regression analyses and logistic regression analyses were used to identify the influence of inflammatory markers and platelet indices in PTC patients. Results are presented as odds ratio (OR) for the predictor variable, $95 \%$ confidence interval (CI) and $p$ value for each variable assumed as a predictor. The overall validity of the model was measured using area under the receiver operating characteristic curve (AUROC) with 95\% CI. The SPSS statistical package for Windows, version 20.0. (IBM Corp. Released 2011. IBM SPSS Statistics for Windows, Version 20.0. Armonk, NY, USA: IBM Corp.), was used to perform all statistical analysis. A $p$ value $<0.05$ indicated statistical significance.

\section{Results}

The clinical and laboratory characteristics of the study population consisting of 234 patients with PTC compared with 108 age-, gender- and BMI-matched control patients with histologic benign thyroid pathology are described in Table 1. PTC patients had significantly higher median levels of TSH (1.21 (1.38) vs. $0.87(1.23) \mu \mathrm{IU} / \mathrm{mL}, p=0.032)$, while FT4 median levels were similar in the two groups $(p=0.515)$. In our patient population, the prevalence of autoimmune thyroiditis was higher in the control group than in the PTC group ( $88.9 \%$ vs. $48.7 \%, \chi^{2}=50.315, p<0.001$ ). The pathological reports data in our PTC patients displayed multifocality in $31.2 \%$, vascular invasion in $12.8 \%$, capsular invasion in $32.1 \%$, extracapsular extension in $20.9 \%$ and lymph node metastases in $15.8 \%$ of cases. Median tumor size was 10 (17) $\mathrm{mm}$ and most patients had either T1 or T2 tumors $(66.7 \%)$ according to the pTNM staging system [24]. 
Table 1. Clinical and laboratory characteristics of the study population.

\begin{tabular}{cccc}
\hline Parameter & $\begin{array}{c}\text { Control Group } \\
\mathbf{N}=\mathbf{1 0 8}\end{array}$ & $\begin{array}{c}\text { PTC } \\
\mathbf{N = \mathbf { 2 3 4 }}\end{array}$ & $\boldsymbol{p}$ \\
\hline Age, mean $\pm \mathrm{SD}$ (years) & $52.07 \pm 12.9$ & $51.54 \pm 13.10$ & 0.726 \\
\hline Sex, N $(\%)$ & & \\
\hline Female & $90(83.3)$ & $198(84.6)$ & 0.762 \\
\hline BMI, mean $\pm \mathrm{SD}\left(\mathrm{kg} / \mathrm{m}^{2}\right)$ & $28.45 \pm 5.79$ & $28.79 \pm 5.7$ & 0.730 \\
\hline TSH, median $(\mathrm{IQR})(\mu \mathrm{IU} / \mathrm{mL})$ & $0.87(1.23)$ & $1.21(1.38)$ & $\mathbf{0 . 0 3 2}$ \\
\hline FT4, median $(\mathrm{IQR})(\mathrm{ng} / \mathrm{dL})$ & $1.14(0.37)$ & $1.08(0.32)$ & 0.515 \\
\hline Autoimmune thyroiditis, $\mathrm{N}(\%)$ & $96(88.9)$ & $114(48.7)$ & $<\mathbf{0 . 0 0 1}$ \\
\hline
\end{tabular}

Abbreviations: BMI, body mass index; FT4, free T4; IQR, interquartile range; PTC, papillary thyroid carcinoma $\mathrm{SD}$, standard deviation; TSH, thyroid stimulating hormone. Bold show statistical significance $(p<0.05)$.

Univariate comparative analysis of the inflammatory markers and platelet indices according to the type of DTC (Table 2) showed that patients with PTC had a significantly higher mean platelet count $\left(263.82 \pm 65.44\right.$ vs. $\left.244.62 \pm 61.59 \times 10^{3} / \mathrm{mm}^{3}, p=0.011\right)$ and PCT levels $(0.28 \pm 0.06$ vs. $0.26 \pm 0.06 \%, p=0.006)$ but similar mean MPV (0.846) and median PDW ( $p=0.967)$ levels when compared with controls. Significantly higher median neutrophil count levels were observed in the PTC group when compared with the control group (4.44 (1.91) vs. $\left.4.05(1.83) \times 10^{3} / \mathrm{mm}^{3}, p=0.022\right)$. Median lymphocyte count $(p=0.976)$, NLR $(p=0.201)$ and PLR $(p=0.727)$ levels were not statistically different compared with controls. Median ESR values did not differ significantly, but patients with both DTC types had significantly higher mean levels of fibrinogen than those in the control group $(338.29 \pm 70.64$ vs. $307.9 \pm 68.01 \mathrm{mg} / \mathrm{dL}, p=0.005$ in PTC group, $357 \pm 62.59$ vs. $307.9 \pm 68.01 \mathrm{mg} / \mathrm{dL}, p=0.029 \mathrm{in}$ FTC group, respectively).

Table 2. Univariate comparative analysis of the inflammatory markers and platelet indices according to the type of DTC.

\begin{tabular}{|c|c|c|c|c|c|c|c|c|}
\hline Parameter & $\begin{array}{l}\text { Control Group } \\
\quad \mathrm{N}=108\end{array}$ & $\begin{array}{c}\text { DTC } \\
\mathrm{N}=249\end{array}$ & $p^{*}$ & $\begin{array}{c}\text { PTC } \\
\mathbf{N}=234 \\
\end{array}$ & $p+$ & $\begin{array}{c}\text { FTC } \\
\mathrm{N}=15\end{array}$ & $p \#$ & $p \pm$ \\
\hline $\begin{array}{l}\text { PLT, mean } \pm \text { SD } \\
\left(\times 10^{3} / \mathrm{mm}^{3}\right)\end{array}$ & $244.62 \pm 61.59$ & $263.12 \pm 65.64$ & 0.013 & $263.82 \pm 65.44$ & 0.011 & $252.2 \pm 70.2$ & 0.661 & 0.507 \\
\hline $\mathrm{PCT}$, mean $\pm \mathrm{SD}(\%)$ & $0.26 \pm 0.06$ & $0.28 \pm 0.06$ & 0.007 & $0.28 \pm 0.06$ & 0.006 & $0.27 \pm 0.07$ & 0.444 & 0.783 \\
\hline $\mathrm{MPV}$, mean $\pm \mathrm{SD}(\mathrm{fL})$ & $10.68 \pm 0.84$ & $10.7 \pm 1.02$ & 0.821 & $10.7 \pm 1.03$ & 0.846 & $10.75 \pm 0.83$ & 0.762 & 0.855 \\
\hline PDW, median (IQR) (\%) & $12.4(2.8)$ & $12.4(3.1)$ & 0.997 & $12.45(3.1)$ & 0.967 & $11.9(1.7)$ & 0.925 & 0.987 \\
\hline $\begin{array}{l}\text { Neutrophil count, } \\
\text { median (IQR) } \\
\left(\times 10^{3} / \mathrm{mm}^{3}\right)\end{array}$ & $4.05(1.83)$ & $4.42(1.89)$ & 0.031 & $4.44(1.91)$ & 0.022 & $4.09(1.81)$ & 0.920 & 0.309 \\
\hline $\begin{array}{l}\text { Lymphocyte count, } \\
\text { median (IQR) } \\
\left(\times 10^{3} / \mathrm{mm}^{3}\right)\end{array}$ & $1.93(0.8)$ & $1.94(0.7)$ & 0.992 & $1.94(0.7)$ & 0.976 & $2.09(0.66)$ & 0.973 & 0.987 \\
\hline NLR, median (IQR) & $2.04(1.11)$ & $2.13(1.26)$ & 0.218 & $2.13(1.27)$ & 0.201 & $2.07(1.16)$ & 0.973 & 0.987 \\
\hline PLR, median (IQR) & $126.07(49.68)$ & $128.86(61.42)$ & 0.936 & $130.58(61.56)$ & 0.727 & $116.29(55.59)$ & 0.285 & 0.294 \\
\hline $\begin{array}{l}\text { ESR, median (IQR) } \\
(\mathrm{mm} / \mathrm{h})\end{array}$ & $13(11)$ & $13(13)$ & 0.743 & $13(12)$ & 0.802 & $12(12)$ & 0.759 & 0.857 \\
\hline $\begin{array}{c}\text { Fibrinogen, mean } \pm \text { SD } \\
(\mathrm{mg} / \mathrm{dL})\end{array}$ & $307.9 \pm 68.01$ & $339.61 \pm 70.08$ & 0.003 & $338.29 \pm 70.64$ & 0.005 & $357 \pm 62.59$ & 0.029 & 0.395 \\
\hline
\end{tabular}

Abbreviations: DTC, differentiated thyroid cancer; ESR, erythrocyte sedimentation rate; FTC, follicular thyroid carcinoma; IQR, interquartile range; MPV, mean platelet volume; NLR, neutrophil-to-lymphocyte ratio; PCT, plateletcrit; PDW, platelet distribution width; PLR, plateletto-lymphocyte ratio; PLT, platelet count; PTC, papillary thyroid carcinoma; SD, standard deviation. $p^{*}$ value comparing DTC patients with controls; $p$ + value comparing PTC patients with controls; $p$ \# value comparing FTC patients with controls; $p \pm$ value comparing FTC with PTC patients. Bold show statistical significance $(p<0.05)$. 
Univariate comparative analysis of the inflammatory markers and platelet indices according to gender revealed that females had significantly higher PLT (268.84 \pm 65.11 vs. $\left.236.19 \pm 60.95 \times 10^{3} / \mathrm{mm}^{3}, p=0.006\right)$, PCT $(0.29 \pm 0.06$ vs. $0.24 \pm 0.05 \%, p<0.001)$ and ESR (13 (12) vs. $6(9) \mathrm{mm} / \mathrm{h}, p=0.005)$, while males had a higher neutrophil count (4.82 (2.11) vs. $\left.4.37(1.87) \times 10^{3} / \mathrm{mm}^{3}, p=0.040\right)$ (Table 3).

Table 3. Univariate comparative analysis of the inflammatory markers and platelet indices according to gender and age in PTC patients.

\begin{tabular}{|c|c|c|c|c|c|c|}
\hline \multirow[b]{2}{*}{ Parameter } & \multicolumn{3}{|c|}{ Gender } & \multicolumn{3}{|c|}{ Age } \\
\hline & $\begin{array}{l}\text { Female } \\
N=198\end{array}$ & $\begin{array}{c}\text { Male } \\
N=36\end{array}$ & $p$ & $\begin{array}{c}<55 \text { Years } \\
N=124\end{array}$ & $\begin{array}{l}>55 \text { Years } \\
N=110\end{array}$ & $p$ \\
\hline $\begin{array}{l}\mathrm{PLT}, \text { mean } \pm \mathrm{SD} \\
\left(\times 10^{3} / \mathrm{mm}^{3}\right)\end{array}$ & $268.84 \pm 65.11$ & $236.19 \pm 60.95$ & 0.006 & $278.96 \pm 62.84$ & $246.75 \pm 64.37$ & $<0.001$ \\
\hline $\mathrm{PCT}$, mean $\pm \mathrm{SD}(\%)$ & $0.29 \pm 0.06$ & $0.24 \pm 0.05$ & $<0.001$ & $0.29 \pm 0.05$ & $0.27 \pm 0.06$ & 0.010 \\
\hline $\mathrm{MPV}$, mean $\pm \mathrm{SD}(\mathrm{fL})$ & $10.73 \pm 1.01$ & $10.52 \pm 1.12$ & 0.253 & $10.56 \pm 0.94$ & $10.85 \pm 1.11$ & 0.032 \\
\hline PDW, median (IQR) (\%) & $12.6(3.15)$ & $12.2(3.4)$ & 0.696 & $12.25(2.85)$ & $13.1(3.43)$ & 0.199 \\
\hline $\begin{array}{l}\text { Neutrophil count, } \\
\text { median (IQR) } \\
\left(\times 10^{3} / \mathrm{mm}^{3}\right)\end{array}$ & $4.37(1.87)$ & $4.82(2.11)$ & 0.040 & $4.27(1.69)$ & $4.86(2.14)$ & 0.068 \\
\hline $\begin{array}{l}\text { Lymphocyte count, } \\
\text { median (IQR) } \\
\left(\times 10^{3} / \mathrm{mm}^{3}\right)\end{array}$ & $1.96(0.69)$ & $1.81(1.05)$ & 0.626 & $1.98(0.67)$ & $1.87(0.74)$ & 0.291 \\
\hline NLR, median (IQR) & $2.12(1.1)$ & $2.81(2.06)$ & 0.587 & $2.06(1.13)$ & $2.43(1.29)$ & 0.013 \\
\hline PLR, median (IQR) & $131.55(56.99)$ & $118.96(93.92)$ & 0.587 & $135.24(64.51)$ & $125.88(62.16)$ & 0.238 \\
\hline $\begin{array}{l}\text { ESR, median (IQR) } \\
(\mathrm{mm} / \mathrm{h})\end{array}$ & $13(12)$ & $6(9)$ & 0.005 & $9(11)$ & $16(13)$ & 0.005 \\
\hline $\begin{array}{l}\text { Fibrinogen, mean } \pm \text { SD } \\
\qquad(\mathrm{mg} / \mathrm{dL})\end{array}$ & $338.25 \pm 68.94$ & $338.5 \pm 82.49$ & 0.988 & $325.09 \pm 65.05$ & $352.43 \pm 74.05$ & 0.019 \\
\hline
\end{tabular}

Abbreviations: ESR, erythrocyte sedimentation rate; IQR, interquartile range; MPV, mean platelet volume; NLR, neutrophil-to-lymphocyte ratio; PCT, plateletcrit; PDW, platelet distribution width; PLR, platelet-to-lymphocyte ratio; PLT, platelet count; PTC, papillary thyroid carcinoma; SD, standard deviation. Bold show statistical significance $(p<0.05)$.

Lining up to the age cutoff chosen by the new TNM classification [24], we divided patients with PTC into two age groups: under and over 55 years old. Univariate comparative analysis of the inflammatory markers and platelet indices according to the age group showed that patients under 55 years old had significantly higher PLT (278.96 \pm 62.84 vs. $\left.246.75 \pm 64.37 \times 10^{3} / \mathrm{mm}^{3}, p<0.001\right)$ and PCT (0.29 \pm 0.05 vs. $\left.0.27 \pm 0.06 \%, p=0.010\right)$, while patients over 55 years old had higher MPV (10.85 \pm 1.11 vs. $10.56 \pm 0.94 \mathrm{fL}, p=0.032)$, NLR (2.43 (1.29) vs. 2.06 (1.13), $p=0.013)$, ESR (16 (13) vs. 9 (11) mm/h, $p=0.005)$ and fibrinogen levels (352.43 \pm 74.05 vs. $325.09 \pm 65.05 \mathrm{mg} / \mathrm{dL}, p=0.019)$ (Table 3).

Univariate comparative analysis of the inflammatory markers and platelet indices according to weight categories in PTC patients did not disclose any significant differences (data not shown).

Pathological report characteristics displayed that PTC patients with lymph node metastases had lower mean fibrinogen levels (314.98 \pm 68.01 vs. $344.11 \pm 70.36 \mathrm{mg} / \mathrm{dL}$, $p=0.047)$ and were younger $(47.3 \pm 12.91$ vs. $52.34 \pm 13.01$ years, $p=0.031)$ than those without lymph node metastases. Furthermore, patients with pathological autoimmune thyroiditis were older (53.6 \pm 11.26 vs. $50.18 \pm 14.06$ years, $p=0.041)$, had higher mean MPV (10.95 \pm 0.96 vs. $10.53 \pm 1.04 \mathrm{fL}, p=0.002)$ and median PDW (13.3 (2.9) vs. $12(3.4) \%$, $p=0.009$ ) values compared with patients without autoimmune thyroiditis. Platelet indices and inflammatory markers were not influenced by the presence of vascular invasion, capsular invasion, extracapsular extension, multifocality or pT classification (Tables 4-6). 
Patients with T3 and T4 tumors were significantly older than those with T1 and T2 tumors $(54.06 \pm 12.94$ vs. $50.28 \pm 13.04$ years, $p=0.037)$.

Table 4. Univariate comparative analysis of inflammatory markers and platelet indices according to pathological report characteristics in PTC patients.

\begin{tabular}{|c|c|c|c|c|c|c|c|c|c|}
\hline \multirow[b]{2}{*}{ Parameter } & \multicolumn{3}{|c|}{ Vascular Invasion } & \multicolumn{3}{|c|}{ Capsular Invasion } & \multicolumn{3}{|c|}{ Extracapsular Extension } \\
\hline & $\begin{array}{l}\text { Present } \\
\mathrm{N}=30\end{array}$ & $\begin{array}{l}\text { Absent } \\
N=204\end{array}$ & $p$ & $\begin{array}{c}\text { Present } \\
N=75\end{array}$ & $\begin{array}{l}\text { Absent } \\
N=159\end{array}$ & $p$ & $\begin{array}{c}\text { Present } \\
N=49\end{array}$ & $\begin{array}{l}\text { Absent } \\
\mathrm{N}=185\end{array}$ & $p$ \\
\hline$\underset{\text { (years) }}{A g e-\text { mean }} \pm \mathrm{SD}$ & $53.77 \pm 16.08$ & $51.22 \pm 12.62$ & 0.411 & $53.07 \pm 13.65$ & $50.82 \pm 12.81$ & 0.222 & $54.33 \pm 12.77$ & $50.81 \pm 13.12$ & 0.094 \\
\hline \multicolumn{10}{|l|}{ Sex N (\%) } \\
\hline Female & $23(76.7)$ & $175(85.8)$ & 0.275 & $61(81.3)$ & $137(86.2)$ & 0.339 & $38(77.6)$ & $160(86.5)$ & 0.123 \\
\hline Male & $7(23.3)$ & $29(14.2)$ & 0.275 & $14(18.7)$ & $22(13.8)$ & 0.339 & $11(22.4)$ & $25(13.5)$ & 0.123 \\
\hline $\begin{array}{c}\mathrm{BMI}-\operatorname{mean} \\
\left(\mathrm{kg} / \mathrm{m}^{2}\right)\end{array}$ & $26.89 \pm 5.53$ & $29.16 \pm 5.69$ & 0.147 & $28.67 \pm 5.61$ & $28.86 \pm 5.8$ & 0.871 & $28.84 \pm 5.88$ & $28.77 \pm 5.68$ & 0.961 \\
\hline $\begin{array}{l}\text { PLT-mean } \pm \text { SD } \\
\left(\times 10^{3} / \mathrm{mm}^{3}\right)\end{array}$ & $263.8 \pm 62.66$ & $263.82 \pm 65.99$ & 0.999 & $268.75 \pm 67.78$ & $261.50 \pm 64.39$ & 0.430 & $273.9 \pm 72.54$ & $261.15 \pm 63.37$ & 0.226 \\
\hline$\underset{(\mathrm{fL})}{\mathrm{MPV} \text {-mean }} \pm \mathrm{SD}$ & $10.58 \pm 1.08$ & $10.71 \pm 1.02$ & 0.505 & $10.6 \pm 1.1$ & $10.74 \pm 0.99$ & 0.328 & $10.44 \pm 1.03$ & $10.77 \pm 1.02$ & 0.051 \\
\hline $\mathrm{PCT}$-mean $\pm \mathrm{SD}(\%)$ & $0.27 \pm 0.05$ & $0.28 \pm 0.06$ & 0.604 & $0.278 \pm 0.059$ & $0.28 \pm 0.06$ & 0.788 & $0.281 \pm 0.07$ & $0.279 \pm 0.006$ & 0.840 \\
\hline $\begin{array}{c}\text { PDW—-median (IQR) } \\
(\%)\end{array}$ & $12.75(2.53)$ & $12.45(3.20)$ & 0.828 & $12.3(3.13)$ & $12.7(3.2)$ & 0.643 & $12(3)$ & $12.7(3.1)$ & 0.302 \\
\hline $\begin{array}{c}\text { Neutrophil } \\
\text { count-median (IQR) } \\
\left(\times 10^{3} / \mathrm{mm}^{3}\right)\end{array}$ & $4.24(2.38)$ & $4.44(1.82)$ & 0.884 & $4.46(2.13)$ & 4.39 (1.9) & 0.928 & $4.76(2.17)$ & $4.39(1.84)$ & 0.478 \\
\hline $\begin{array}{c}\text { Lymphocyte } \\
\text { count-median (IQR) } \\
\left(\times 10^{3} / \mathrm{mm}^{3}\right)\end{array}$ & $1.78(0.72)$ & $1.97(0.70)$ & 0.087 & $1.84(0.93)$ & $1.97(0.65)$ & 0.190 & $1.85(0.9)$ & $1.97(0.67)$ & 0.223 \\
\hline NLR—median (IQR) & $2.29(2.21)$ & $2.13(1.14)$ & 0.845 & $2.2(1.84)$ & $2.11(1.03)$ & 0.889 & $2.31(1.86)$ & $2.13(1.07)$ & 0.872 \\
\hline PLR—median (IQR) & $159.46(89.74)$ & $128.42(54.60)$ & 0.328 & $131.47(62.9)$ & $127.98(61.68)$ & 0.889 & $142.39(62.58)$ & $127.59(62.17)$ & 0.335 \\
\hline $\begin{array}{c}\text { ESR—median }(\mathrm{IQR}) \\
(\mathrm{mm} / \mathrm{h})\end{array}$ & $13(21)$ & $13(12)$ & 0.791 & $12.5(15)$ & $13(12)$ & 0.999 & $13(17)$ & $12.5(12)$ & 0.976 \\
\hline $\begin{array}{l}\text { Fibrinogen-mean } \pm \\
\mathrm{SD}(\mathrm{mg} / \mathrm{dL})\end{array}$ & $319.18 \pm 73.35$ & $341.34 \pm 70.01$ & 0.194 & $324.82 \pm 69.79$ & $344.95 \pm 70.46$ & 0.107 & $327.66 \pm 67.71$ & $341.3 \pm 71.45$ & 0.337 \\
\hline
\end{tabular}

Abbreviations: BMI, body mass index; ESR, erythrocyte sedimentation rate; IQR, interquartile range; MPV, mean platelet volume; NLR, neutrophil-to-lymphocyte ratio; PCT, plateletcrit; PDW, platelet distribution width; PLR, platelet-to-lymphocyte ratio; PLT, platelet count; PTC, papillary thyroid carcinoma; SD, standard deviation.

Overall, preoperative values for platelet indices (platelets count, MPV, PCT, PDW) ESR, neutrophil count, lymphocyte count, NLR and PLR were similar to the postoperative determinations in PTC patients (Table 7). However, when analyzing the PTC patients based on the pathological report findings (multifocality, capsular invasion, vascular invasion, extracapsular extension, lymph nodes metastasis, autoimmune thyroiditis, pT stage; data not shown) our data exhibit lower median ESR levels before thyroidectomy in patients with no capsular invasion (12 (10) vs. $14(14) \mathrm{mm} / \mathrm{h}, p=0.043)$, and no extracapsular extension, respectively (12 (13) vs. $14(14) \mathrm{mm} / \mathrm{h}, p=0.030)$. When it comes to platelet indices, we found lower mean PLT before surgery in PTC patients with vascular invasion $\left(233.63 \pm 62.06\right.$ vs. $\left.262.13 \pm 55.97 \times 10^{3} / \mathrm{mm}^{3}, p=0.013\right)$.

Simple linear regression analysis showed that, in PTC patients, higher platelet-to-lymphocyte ratio is associated with larger cancer diameter (coefficient $=0.042,95 \% \mathrm{CI}=0.002-0.083, p=0.042$ ).

Multivariate binary logistic regression analysis was performed to ascertain the effects of preoperative platelet indices and inflammatory markers on the likelihood that the patients have PTC. The logistic regression model was statistically significant, $\chi^{2}(6)=13.559$, $p=0.035$. The model explained $10.5 \%$ (Nagelkerke R2) of the variance in PTC and correctly classified $67.8 \%$ of cases. Fibrinogen $(\mathrm{OR}=1.007,95 \% \mathrm{CI}=1.001-1.013, p=0.026)$ and platelet count ( $\mathrm{OR}=1.008,95 \% \mathrm{CI}=1.001-1.014, p=0.018)$ were independent predictors of the presence of PTC. There was a good capacity of fibrinogen (AUROC $=0.602,95 \%$ CI: $0.518-0.685, p=0.02$; cut-off $=327.5 \mathrm{mg} / \mathrm{dL}, \mathrm{Se}=53.8 \%, \mathrm{Sp}=62.9 \%$ (Figure $2 \mathrm{a})$ and 
platelet count $\left(\right.$ AUROC $=0.584,95 \%$ CI: 0.519-0.649, $p=0.012$; cut-off $=223.5 \times 10^{3} / \mathrm{mm}^{3}$, $\mathrm{Se}=73.1 \%, \mathrm{Sp}=42.6 \%$ ) (Figure $2 \mathrm{~b}$ ) to predict the presence of PTC.

Table 5. Univariate comparative analysis of inflammatory markers and platelet indices according to pathological report characteristics in PTC patients.

\begin{tabular}{|c|c|c|c|c|c|c|c|c|c|}
\hline \multirow[b]{2}{*}{ Parameter } & \multicolumn{3}{|c|}{ Multifocality } & \multicolumn{3}{|c|}{ Lymph Node Metastases } & \multicolumn{3}{|c|}{ Autoimmune Thyroiditis (HP) } \\
\hline & $\begin{array}{c}\text { Present } \\
N=73\end{array}$ & $\begin{array}{l}\text { Absent } \\
N=161\end{array}$ & $p$ & $\begin{array}{c}\text { Present } \\
N=37\end{array}$ & $\begin{array}{l}\text { Absent } \\
N=197\end{array}$ & $p$ & $\begin{array}{c}\text { Present } \\
N=93\end{array}$ & $\begin{array}{l}\text { Absent } \\
\mathrm{N}=141\end{array}$ & $p$ \\
\hline$\underset{\text { (years) }}{\text { Age-mean }} \pm \mathrm{SD}$ & $50.26 \pm 14.17$ & $52.12 \pm 12.56$ & 0.314 & $47.3 \pm 12.91$ & $52.34 \pm 13.01$ & 0.031 & $53.6 \pm 11.26$ & $50.18 \pm 14.06$ & 0.041 \\
\hline \multicolumn{10}{|l|}{$\operatorname{Sex} N(\%)$} \\
\hline Female & $61(83.6)$ & $137(85.1)$ & 0.764 & $28(75.7)$ & $170(86.3)$ & 0.100 & $83(89.2)$ & $115(81.6)$ & 0.111 \\
\hline Male & $12(16.4)$ & $24(14.9)$ & 0.764 & $9(24.3)$ & $27(13.7)$ & 0.100 & $10(10.8)$ & $26(18.4)$ & 0.111 \\
\hline$\underset{\left(\mathrm{kg} / \mathrm{m}^{2}\right)}{\mathrm{BMI}-\operatorname{mean}} \pm \mathrm{SD}$ & $29.27 \pm 6.58$ & $28.63 \pm 5.41$ & 0.630 & $29.31 \pm 6.99$ & $28.72 \pm 5.54$ & 0.739 & $27.82 \pm 4.99$ & $29.53 \pm 6.13$ & 0.139 \\
\hline $\begin{array}{l}\text { PLT-mean } \pm \text { SD } \\
\left(\times 10^{3} / \mathrm{mm}^{3}\right)\end{array}$ & $261.97 \pm 66.33$ & $264.66 \pm 65.22$ & 0.772 & $270.49 \pm 62.78$ & $262.57 \pm 66$ & 0.501 & $254.47 \pm 68.75$ & $269.99 \pm 62.65$ & 0.076 \\
\hline$\underset{(\mathrm{fL})}{\mathrm{MPV} \text {-mean }} \pm \mathrm{SD}$ & $10.78 \pm 1.1$ & $10.66 \pm 1$ & 0.425 & $10.4 \pm 1.06$ & $10.75 \pm 1.01$ & 0.057 & $10.95 \pm 0.96$ & $10.53 \pm 1.04$ & 0.002 \\
\hline $\mathrm{PCT}$-mean $\pm \mathrm{SD}(\%)$ & $0.27 \pm 0.055$ & $0.28 \pm 0.062$ & 0.350 & $0.278 \pm 0.057$ & $0.28 \pm 0.06$ & 0.847 & $0.27 \pm 0.064$ & $0.28 \pm 0.057$ & 0.359 \\
\hline $\begin{array}{c}\text { PDW—-median (IQR) } \\
(\%)\end{array}$ & $12.75(3.55)$ & $12.4(2.97)$ & 0.440 & $11.8(2.9)$ & $12.8(3.1)$ & 0.137 & $13.3(2.9)$ & $12(3.4)$ & 0.009 \\
\hline $\begin{array}{c}\text { Neutrophil } \\
\text { count-median (IQR) } \\
\left(\times 10^{3} / \mathrm{mm}^{3}\right)\end{array}$ & $4.33(1.62)$ & $4.57(2.04)$ & 0.186 & $4.42(2.12)$ & $4.44(1.86)$ & 0.955 & $4.33(1.97)$ & $4.46(1.9)$ & 0.669 \\
\hline $\begin{array}{c}\text { Lymphocyte } \\
\text { count-median (IQR) } \\
\left(\times 10^{3} / \mathrm{mm}^{3}\right)\end{array}$ & $1.97(0.88)$ & $1.93(0.68)$ & 0.711 & $1.84(0.57)$ & $1.97(0.72)$ & 0.083 & $1.96(0.6)$ & $1.91(0.74)$ & 0.915 \\
\hline NLR—median (IQR) & $2.09(1.09)$ & $2.16(1.28)$ & 0.778 & $2.31(1.97)$ & $2.13(1.12)$ & 0.858 & $2.09(1.25)$ & $2.21(1.28)$ & 0.593 \\
\hline PLR—median (IQR) & $130.27(62.66)$ & $131.63(62.51)$ & 0.888 & $142.39(62.76)$ & $127.59(64.02)$ & 0.282 & $125.56(52.7)$ & $135.05(64.71)$ & 0.182 \\
\hline $\begin{array}{l}\text { ESR—median }(\mathrm{IQR}) \\
(\mathrm{mm} / \mathrm{h})\end{array}$ & $13(12)$ & $12.5(14)$ & 0.750 & $9(9)$ & $13(14)$ & 0.138 & $13(14)$ & $12(12)$ & 0.843 \\
\hline $\begin{array}{l}\text { Fibrinogen-mean } \pm \\
\text { SD }(\mathrm{mg} / \mathrm{dL})\end{array}$ & $324.22 \pm 63.58$ & $345.46 \pm 73.26$ & 0.087 & $314.98 \pm 68.01$ & $344.11 \pm 70.36$ & 0.047 & $346.43 \pm 68.12$ & $332.7 \pm 72.18$ & 0.251 \\
\hline
\end{tabular}

Abbreviations: BMI, body mass index; ESR, erythrocyte sedimentation rate; IQR, interquartile range; MPV, mean platelet volume; NLR, neutrophil-to-lymphocyte ratio; PCT, plateletcrit; PDW, platelet distribution width; PLR, platelet-to-lymphocyte ratio; PLT, platelet count; PTC, papillary thyroid carcinoma; SD, standard deviation. Put in bold the $p$ values that show statistical significance $(p<0.05)$.

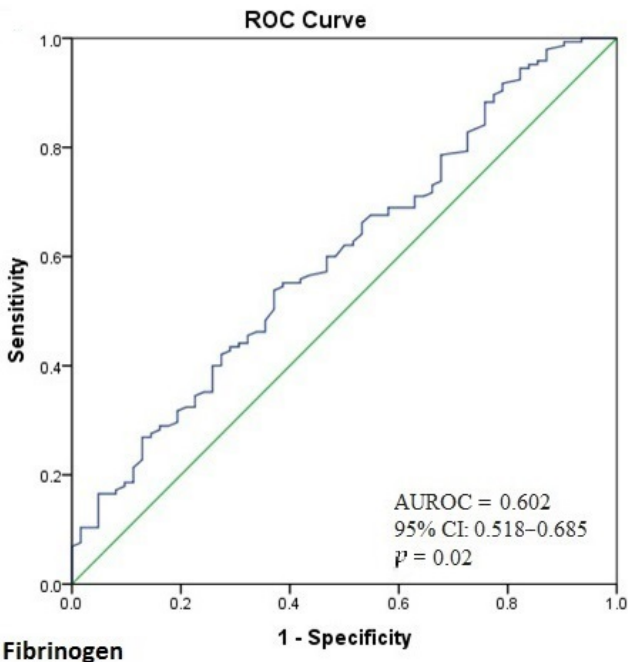

(a)

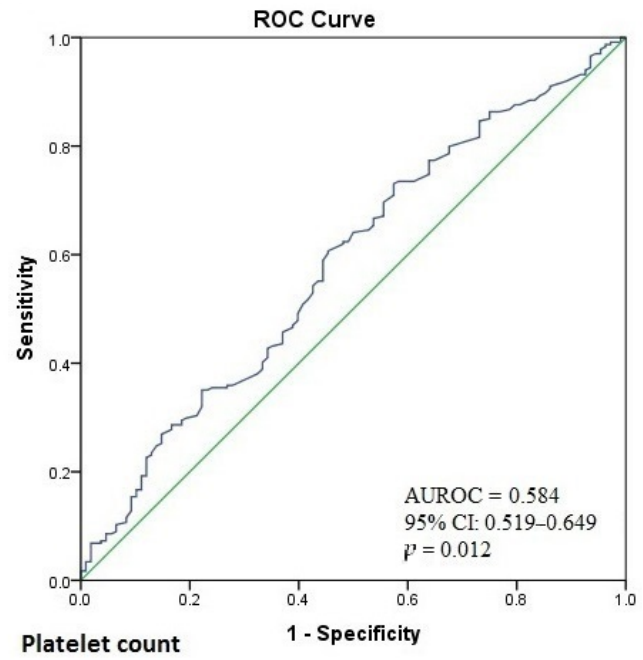

(b)

Figure 2. (a) Receiver operating characteristic (ROC) curve for the predictive value of fibrinogen on the presence of PTC; (b) Receiver operating characteristic (ROC) curve for the predictive value of platelet count on the presence of PTC. 
Table 6. Univariate comparative analysis of the inflammatory markers and platelet indices according to pathological $\mathrm{pT}$ classification.

\begin{tabular}{|c|c|c|c|c|c|c|c|c|}
\hline \multirow[b]{2}{*}{ Parameter } & \multicolumn{8}{|c|}{ TNM Staging-pT Classification } \\
\hline & $\begin{array}{c}\mathrm{T} 1 \\
\mathrm{~N}=128\end{array}$ & $\begin{array}{c}\mathrm{T} 2 \\
\mathrm{~N}=28\end{array}$ & $\begin{array}{c}\mathrm{T} 3 \\
\mathrm{~N}=73\end{array}$ & $\begin{array}{c}\mathrm{T} 4 \\
\mathrm{~N}=5\end{array}$ & $p$ & $\begin{array}{c}\text { T1-T2 } \\
\mathbf{N}=156\end{array}$ & $\begin{array}{l}\text { T3-T4 } \\
\mathrm{N}=78\end{array}$ & $p$ \\
\hline Age-mean \pm SD (years) & $51.54 \pm 11.95$ & $44.29 \pm 15.65$ & $54.19 \pm 12.78$ & $52.2 \pm 16.57$ & 0.008 & $50.28 \pm 13.04$ & $54.06 \pm 12.94$ & 0.037 \\
\hline $\mathrm{BMI}-$ mean $\pm \mathrm{SD}\left(\mathrm{kg} / \mathrm{m}^{2}\right)$ & $28.89 \pm 5.61$ & $27.59 \pm 4.82$ & $29.36 \pm 6.13$ & $24.08 \pm 6.86$ & 0.493 & $28.53 \pm 5.34$ & $29.12 \pm 6.18$ & 0.612 \\
\hline $\begin{array}{l}\text { PLT, mean } \pm \text { SD } \\
\left(\times 10^{3} / \mathrm{mm}^{3}\right)\end{array}$ & $256.89 \pm 63.72$ & $274.25 \pm 65.49$ & $269.04 \pm 67.51$ & $309.8 \pm 77.62$ & 0.171 & $259.9 \pm 63.78$ & $271.65 \pm 68.38$ & 0.196 \\
\hline $\mathrm{PCT}$, mean $\pm \mathrm{SD}(\%)$ & $0.279 \pm 0.061$ & $0.287 \pm 0.063$ & $0.277 \pm 0.058$ & $0.31 \pm 0.087$ & 0.744 & $0.28 \pm 0.061$ & $0.276 \pm 0.059$ & 0.849 \\
\hline $\mathrm{MPV}$, mean $\pm \mathrm{SD}(\mathrm{fL})$ & $10.81 \pm 0.97$ & $10.6 \pm 1$ & $10.58 \pm 1.12$ & $10.02 \pm 1.09$ & 0.174 & $10.78 \pm 0.97$ & $10.54 \pm 1.12$ & 0.100 \\
\hline PDW, median (IQR) (\%) & $12.8(3.1)$ & $12.3(3.65)$ & $12.2(3.55)$ & $13.4(-)$ & 0.491 & $12.7(3.1)$ & $12.2(3.5)$ & 0.539 \\
\hline $\begin{array}{l}\text { Neutrophil count, median } \\
(\mathrm{IQR})\left(\times 10^{3} / \mathrm{mm}^{3}\right)\end{array}$ & $4.48(1.9)$ & $4.2(1.78)$ & $4.46(2.16)$ & $5.04(3.56)$ & 0.319 & $4.43(1.88)$ & $4.51(2.16)$ & 0.817 \\
\hline $\begin{array}{l}\text { Lymphocyte count, median } \\
\text { (IQR) }\left(\times 10^{3} / \mathrm{mm}^{3}\right)\end{array}$ & $1.97(0.68)$ & $2.01(0.72)$ & $1.85(0.81)$ & $1.66(1.53)$ & 0.683 & $1.97(0.68)$ & $1.85(0.88)$ & 0.248 \\
\hline NLR, median (IQR) & $2.16(1.05)$ & $1.86(1.16)$ & $2.13(1.67)$ & $2.98(3.35)$ & 0.149 & $2.11(1.05)$ & $2.17(1.72)$ & 0.890 \\
\hline PLR, median (IQR) & $125.01(55.25)$ & $126.65(85.74)$ & $133.83(49.73)$ & $186.92(107.21)$ & 0.362 & $125.82(62.7)$ & $136.24(56.35)$ & 0.332 \\
\hline ESR, median (IQR) (mm/h) & $13(13)$ & $11.5(12)$ & $13(18)$ & $7(23)$ & 0.439 & $12(12)$ & $13(18)$ & 0.952 \\
\hline $\begin{array}{l}\text { Fibrinogen, mean } \pm S D \\
\qquad(\mathrm{mg} / \mathrm{dL})\end{array}$ & $345.35 \pm 70.04$ & $334.67 \pm 64.53$ & $329.87 \pm 75.52$ & $308.8 \pm 67.95$ & 0.518 & $343.54 \pm 68.5$ & $327.68 \pm 74.37$ & 0.204 \\
\hline
\end{tabular}

Abbreviations: BMI, body mass index; ESR, erythrocyte sedimentation rate; IQR, interquartile range; MPV, mean platelet volume; NLR, neutrophil-to-lymphocyte ratio; PCT, plateletcrit; PDW, platelet distribution width; PLR, platelet-to-lymphocyte ratio; PLT, platelet count; $\mathrm{SD}$, standard deviation.

Table 7. Univariate comparative analysis of pre- and postoperative levels of inflammatory markers and platelet indices in PTC patients.

\begin{tabular}{cccc}
\hline Parameter & $\begin{array}{c}\text { Preoperative } \\
\mathbf{N}=\mathbf{2 3 4}\end{array}$ & $\begin{array}{c}\text { Postoperative } \\
\mathbf{N}=\mathbf{6 3}\end{array}$ & $p$ \\
\hline PLT, mean $\pm \mathrm{SD}\left(\times 10^{3} / \mathrm{mm}^{3}\right)$ & $266.35 \pm 60.86$ & $265.14 \pm 69.39$ & 0.865 \\
\hline MPV, mean $\pm \mathrm{SD}(\mathrm{fL})$ & $10.57 \pm 1$ & $10.51 \pm 1.04$ & 0.382 \\
\hline PCT, mean $\pm \mathrm{SD}(\%)$ & $0.277 \pm 0.054$ & $0.275 \pm 0.062$ & 0.841 \\
\hline PDW, median $(\mathrm{IQR})(\%)$ & $12.4(3.5)$ & $12.5(2.85)$ & 0.963 \\
\hline ESR, median $(\mathrm{IQR})(\mathrm{mm} / \mathrm{h})$ & $12(14)$ & $14(14)$ & 0.088 \\
\hline Lymphocyte count, median $(\mathrm{IQR})\left(\times 10^{3} / \mathrm{mm}^{3}\right)$ & $1.85(0.76)$ & $1.83(0.71)$ & 0.354 \\
\hline NLR, median $(\mathrm{IQR})$ & $2.49(1.33)$ & $2.27(1.28)$ & 0.653 \\
\hline PLR, median $(\mathrm{IQR})$ & $138.93(51.38)$ & $143.48(78.72)$ & 0.536 \\
\hline
\end{tabular}

Abbreviations: ESR, erythrocyte sedimentation rate; IQR, interquartile range; MPV, mean platelet volume; NLR, neutrophil-to-lymphocyte ratio; PCT, plateletcrit; PDW, platelet distribution width; PLR, platelet-to-lymphocyte ratio; PLT, platelet count; PTC, papillary thyroid carcinoma; SD, standard deviation.

\section{Discussion}

In this retrospective study we analyzed the preoperatory inflammatory markers and platelet indices in 234 PTC patients compared with an age, gender and BMI control group of 108 patients with benign thyroid pathology. Furthermore, we investigated the relationship between inflammatory markers and platelet indices and pathological features of PTC and we compared pre- and postoperative inflammatory markers and platelet indices in PTC patients.

Xenograft experiments and transgenic mouse models demonstrated that platelet activation and platelet-cancer cell interaction are crucial for cancer development and metastasis. Growth factors, metabolites and microRNA released by activated platelets induce epithelial-to-mesenchymal transition and enhance cancer cell stemness, which is crucial for cancer cell colonization at the distant organs. Direct or indirect interaction 
of platelets induces cancer cell plasticity and enhances survival and extravasation of circulating cancer cells during dissemination. On the other hand, in vivo and in vitro experiments demonstrate that cancer cells induce platelet activation and aggregation, subsequently elevating the risk of thrombosis, suggesting that platelet-cancer interaction is bidirectional [9]. Furthermore, platelets interactions with cancer cells and the tumor microenvironment are very complex and seem to have dual behaviors: pro and anticancerous, with the procancerogenic effect out-numbering the anti-cancerous effects [25]. Targeting platelet-cancer cell interaction may be a potential strategy for reducing both cancer metastasis and cancer-associated thrombosis [9].

Given their broad accessibility, low cost and high reproducibility rates, platelet count and their indices-MPV, PDW and PCT—have been used as inflammatory markers in cancer patients and the subject of numerous studies in different types of cancer, but there are scarce and conflicting data surrounding their role as biomarkers in thyroid cancer, possibly due to the small size population in the previous studies and the excellent prognosis and high survival rates.

Our PTC patients had higher PLT and PCT when compared with controls, a finding that can be explained by the previously described overproduction of interleukin-6 in malignancies, which stimulates the production of thrombopoietin and leads to megakaryocyte proliferation [26]. To the best of our knowledge, we are the first to show that PLT and fibrinogen are independent predictors for the presence of PTC. Using ROC analysis, we found a cutoff value of $327.5 \mathrm{mg} / \mathrm{dL}$ for fibrinogen to predict the presence of PTC with a sensitivity of $53.8 \%$ and a specificity of $62.9 \%$ and a cutoff value of $223.5 \times 10^{3} / \mathrm{mm}^{3}$ for platelet number to predict the presence of PTC with a sensitivity of $73.1 \%$ and a specificity of $42.6 \%$. Recently, Mizrak and Kucuk [27] also reported higher platelet count in PTC patients. Additionally, Dincel and Bayratkar noted similar results regarding PCT, but they found no significant difference in PLT between PTC and benign multinodular goiter patients [17]. Supporting our data, Jianyong et al. found that hyperfibrinogenemia is associated with advanced tumor stage and a high rate of recurrence, which enabled them to elaborate a nomogram that can be used to predict recurrence of papillary thyroid carcinoma [28].

Baldane et al. [16] reported that PTC patients have higher MPV values, which decrease after thyroidectomy, and their results were replicated by Ozmen et al. [29]. On the other hand, Yu et al. found lower MPV values in patients with thyroid cancer when compared with controls, but their study group included both DTC and MTC [30]. Our data showed no significant difference in MPV between PTC patients and controls. Additionally, we found no significant difference between preoperative and postoperative values for platelet indices and inflammatory markers, a finding that could be explained by the short time between surgery and the first follow-up visit, or by the connate inflammatory profile in cancer patients.

PDW also assesses the platelet volume, indicating their variation in size. Although often overlooked in everyday clinical practice, Xia et al. showed in their meta-analysis that a high PDW level is a prognostic factor for cancer and is associated with lymph node metastasis [10], while Dincel and Bayraktar obtained significantly lower PDW values in PTC patients when compared with controls [17]. Nevertheless, we could not find any significant difference in PDW levels among our study groups.

NLR and PLR have been widely studied as biomarkers in inflammatory conditions and cancer, but with inconsistent results in thyroid malignancies. A high number of neutrophils suppresses the immune system by inhibiting lymphocytes, activated $T$ cells and natural killer cells in their cytolytic activity. On the other hand, lymphocytes and increased tumoral lymphocytic infiltration have been associated with better outcomes in cancer patients [31]. Seretis et al. were the first to show that NLR may predict the presence of PTC [11]. Subsequent studies showed higher NLR in PTC patients [22,32] and association with tumor size [14,33], extrathyroidal extension [33], lymph node metastases [34] or cancer recurrence [13]. Furthermore, a recent meta-analysis published by Feng et al. 
underlines the role of NLR as a biomarker for the prediction of tumor growth, metastasis and prognosis in patients with thyroid cancer [35]. Even though we did find a higher neutrophil count in the PTC group, we were not able to replicate the results of the previously mentioned studies. Our data did not reveal PLR as a useful marker in differentiating between PTC and benign thyroid disorders. However, consistent with the findings of Kim et al., who reported higher PLR in patients with tumors larger than $1 \mathrm{~cm}$, in our PTC patients, higher PLR was associated with larger tumor size [36]. Looking further at the pathological reports and trying to find whether platelet indices or inflammatory markers can predict an aggressive tumor behavior, we surprisingly found lower fibrinogen levels in PTC patients with lymph node metastases-data that contradicts recent research on esophageal and gastric cancers [37,38]. However, we were able to replicate the results obtained by Wen et al. [19] regarding PDW and MPV, which were higher in patients with pathological autoimmune thyroiditis. Even though the presence of autoimmune thyroiditis was significantly higher in the control group, it was still highly prevalent in the PTC group as well, with almost half of the patients diagnosed with autoimmune thyroiditis based on thyroid antibody positivity and/or the pathological diagnosis. Published data show that up to one-third of PTC cases are associated with autoimmune thyroiditis [39]. For this reason, we chose to include all patients in the study, regardless of the presence of thyroid autoimmunity, in order to replicate the PTC cases that we evaluate in the clinical practice. While Machairas et al. [21] showed higher MPV in multifocal PTC and higher PLT, PCT and PLR in cases of extrathyroidal extension, platelet indices and inflammation did not differ depending on the presence of vascular invasion, capsular invasion, extracapsular extension, multifocality or pT classification in our study.

The strengths of this study are the important sample size of our thyroid cancer patients, the well-matched controls and the availability of pre- and postoperative data, as well as the detailed pathological reports. The main limitations are the retrospective nature of the study, which made it difficult to exclude other major factors that could have influenced fibrinogen levels and platelet indices, such as diabetes, smoking, infections, inflammatory diseases, iron deficiency and the use of antiplatelet drugs [40-42] and the lack of other inflammatory markers such as CRP (C-reactive protein), TNF- $\alpha$ (tumor necrosis factor- $\alpha$ ), interleukin-6. The relationship between platelet indices, inflammatory markers and prognosis in PTC was not the subject of this paper, but it will be analyzed in a subsequent study as we gather more follow-up data.

\section{Conclusions}

Thyroid carcinomas are the most common type of endocrine malignancies, and their incidence has increased in the last decades due to widespread use of high-resolution ultrasonography. In the attempt to find markers that differentiate between benign and malignant thyroid nodules and help select appropriate treatments, our data show that fibrinogen and platelet count could be promising, inexpensive, independent predictors for the presence of PTC when compared with benign thyroid disorders.

Author Contributions: Conceptualization, writing-review and editing, S.M.; data curation, T.M., O.E., O.I. and A.C.; writing-original draft preparation, A.S., C.B., A.M., C.G. and F.A.; supervision, S.F. All authors have read and agreed to the published version of the manuscript.

Funding: This research received no external funding.

Institutional Review Board Statement: The study was conducted according to the guidelines of the Declaration of Helsinki, and the study was approved by the Ethics Committee of Elias Hospital $(1489 / 18.02 .2021)$.

Informed Consent Statement: Informed consent was obtained from all subjects involved in the study.

Data Availability Statement: The data presented in this study are available on request from the corresponding author.

Acknowledgments: Not applicable. 
Conflicts of Interest: The authors declare no conflict of interest.

\section{References}

1. Pellegriti, G.; Frasca, F.; Regalbuto, C.; Squatrito, S.; Vigneri, R. Worldwide increasing incidence of thyroid cancer: Update on epidemiology and risk factors. J. Cancer Epidemiol. 2013, 2013, 965212. [CrossRef] [PubMed]

2. Haugen, B.R.; Alexander, E.K.; Bible, K.C.; Doherty, G.M.; Mandel, S.J.; Nikiforov, Y.E.; Pacini, F.; Randolph, G.W.; Sawka, A.M.; Schlumberger, M.; et al. 2015 American Thyroid Association Management Guidelines for Adult Patients with Thyroid Nodules and Differentiated Thyroid Cancer: The American Thyroid Association Guidelines Task Force on Thyroid Nodules and Differentiated Thyroid Cancer. Thyroid 2016, 26, 1-133. [CrossRef] [PubMed]

3. Pagano, L.; Mele, C.; Sama, M.T.; Zavattaro, M.; Caputo, M.; De Marchi, L.; Paggi, S.; Prodam, F.; Aimaretti, G.; Marzullo, P. Thyroid cancer phenotypes in relation to inflammation and autoimmunity. Front. Biosci. 2018, 23, 2267-2282. [CrossRef]

4. Liotti, F.; Visciano, C.; Melillo, R.M. Inflammation in thyroid oncogenesis. Am. J. Cancer Res. 2012, 2, 286-297. [PubMed]

5. Gonda, T.A.; Tu, S.; Wang, T.C. Chronic inflammation, the tumor microenvironment and carcinogenesis. Cell Cycle 2009, 8, 2005-2013. [CrossRef] [PubMed]

6. Coussens, L.M.; Werb, Z. Inflammation and Cancer. Nature 2002, 420, 860-867. [CrossRef] [PubMed]

7. Vakkila, J.; Lotze, M.T. Inflammation and necrosis promote tumour growth. Nat. Rev. Immunol. 2004, 4, 641-648. [CrossRef] [PubMed]

8. Kim, S.; Takahashi, H.; Lin, W.W.; Descargues, P.; Grivennikov, S.; Kim, Y.; Luo, J.L.; Karin, M. Carcinoma-produced factors activate myeloid cells through TLR2 to stimulate metastasis. Nature 2009, 457, 102-106. [CrossRef] [PubMed]

9. Wang, S.; Li, Z.; Xu, R. Human Cancer and Platelet Interaction, a Potential Therapeutic Target. Int. J. Mol. Sci. 2018, 19, 1246. [CrossRef]

10. Xia, W.; Chen, W.; Tu, J.; Ni, C.; Meng, K. Prognostic Value and Clinicopathologic Features of Platelet Distribution Width in Cancer: A Meta-Analysis. Med. Sci. Monit. 2018, 24, 7130-7136. [CrossRef] [PubMed]

11. Seretis, C.; Gourgiotis, S.; Gemenetzis, G.; Seretis, F.; Lagoudianakis, E.; Dimitrakopoulos, G. The significance of neutrophil/ lymphocyte ratio as a possible marker of underlying papillary microcarcinomas in thyroidal goiters: A pilot study. Am. J. Surg. 2013, 205, 691-696. [CrossRef] [PubMed]

12. Gong, W.; Yang, S.; Yang, X.; Guo, F. Blood preoperative neutrophil-to-lymphocyte ratio is correlated with TNM stage in patients with papillary thyroid cancer. Clinics 2016, 71, 311-314. [CrossRef]

13. Offi, C.; Romano, R.M.; Cangiano, A.; Candela, G.; Docimo, G. Clinical significance of neutrophil-to-lymphocyte ratio, lymphocyteto-monocyte ratio, platelet-to-lymphocyte ratio and prognostic nutritional index in low-risk differentiated thyroid carcinoma. Acta Otorhinolaryngol. Ital. 2021, 41, 31-38. [CrossRef] [PubMed]

14. Cheong, T.Y.; Hong, S.D.; Jung, K.W.; So, Y.K. The diagnostic predictive value of neutrophil-to-lymphocyte ratio in thyroid cancer adjusted for tumor size. PLoS ONE 2021, 16, e0251446. [CrossRef] [PubMed]

15. Bayhan, Z.; Zeren, S.; Ozbay, I.; Kahraman, C.; Yaylak, F.; Tiryaki, C.; Ekici, M. Mean Platelet Volume as a Biomarker for Thyroid Carcinoma. Int. Surg. 2016, 101, 50-53. [CrossRef] [PubMed]

16. Baldane, S.; Ipekci, S.H.; Sozen, M.; Kebapcilar, L. Mean platelet volume could be a possible biomarker for papillary thyroid carcinomas. Asian Pac. J. Cancer Prev. 2015, 16, 2671-2674. [CrossRef] [PubMed]

17. Dincel, O.; Bayraktar, C. Evaluation of platelet indices as a useful marker in papillary thyroid carcinoma. Bratislavske Lekarske Listy 2017, 118, 153-155. [CrossRef] [PubMed]

18. Li, N.; Fu, S.; Cui, M.M.; Niu, Y.; Li, B.; Liu, Z.P.; Liu, T.; Wang, R.T. Platelet Distribution Width and Serum Albumin Levels for Discrimination of Thyroid Cancer from Benign Thyroid Nodules. Asian Pac. J. Cancer Prev. 2017, 18, 1773-1777. [CrossRef] [PubMed]

19. Wen, W.; Wu, P.; Li, J.; Wang, H.; Sun, J.; Chen, H. Predictive values of the selected inflammatory index in elderly patients with papillary thyroid cancer. J. Transl. Med. 2018, 16, 261. [CrossRef] [PubMed]

20. Yaylaci, S.; Tosun, O.; Sahin, O.; Genc, A.B.; Aydın, E.; Demiral, G.; Karahalil, F.; Olt, S.; Ergenç, H.; Varim, C. Lack of Variation in Inflammatory Hematological Parameters between Benign Nodular Goiter and Papillary Thyroid Cancer. Asian Pac. J. Cancer Prev. 2016, 17, 2321-2323. [CrossRef] [PubMed]

21. Machairas, N.; Kostakis, I.D.; Prodromidou, A.; Stamopoulos, P.; Feretis, T.; Garoufalia, Z.; Damaskos, C.; Tsourouflis, G.; Kouraklis, G. Trends in white blood cell and platelet indices in a comparison of patients with papillary thyroid carcinoma and multinodular goiter do not permit differentiation between the conditions. Endocr. Res. 2017, 42, 311-317. [CrossRef] [PubMed]

22. Manatakis, D.K.; Tseleni-Balafouta, S.; Tzelves, L.; Balalis, D.; Tzortzopoulou, A.; Korkolis, D.P.; Sakorafas, G.H.; Gontikakis, E.; Plataniotis, G. Diagnostic Accuracy of Preoperative Neutrophil-to-Lymphocyte and Platelet-to-Lymphocyte Ratios in Detecting Occult Papillary Thyroid Microcarcinomas in Benign Multinodular Goitres. J. Thyroid Res. 2018, 2018, 3470429. [CrossRef] [PubMed]

23. Nikiforov, Y.E.; Seethala, R.R.; Tallini, G.; Baloch, Z.W.; Basolo, F.; Thompson, L.D.; Barletta, J.A.; Wenig, B.M.; Al Ghuzlan, A.; Kakudo, K.; et al. Nomenclature Revision for Encapsulated Follicular Variant of Papillary Thyroid Carcinoma: A Paradigm Shift to Reduce Overtreatment of Indolent Tumors. JAMA Oncol. 2016, 2, 1023-1029. [CrossRef] [PubMed] 
24. Tuttle, M.; Morris, L.F.; Haugen, B.; Shah, J.; Sosa, J.A.; Rohren, E.; Subramaniam, R.M.; Hunt, J.L.; Perrier, N.D. Thyroiddifferentiated and anaplastic carcinoma. In AJCC Cancer Staging Manual, 8th ed.; Amin, M.B., Edge, S.B., Greene, F., Byrd, D., Brookland, R.K., Washington, M.K., Gershenwald, J.E., Compton, C.C., Hess, K.R., Sullivan, D.C., et al., Eds.; Springer International Publishing: New York, NY, USA, 2017; pp. 873-901. ISBN 9783319406176.

25. Palacios-Acedo, A.L.; Mège, D.; Crescence, L.; Dignat-George, F.; Dubois, C.; Panicot-Dubois, L. Platelets, Thrombo-Inflammation, and Cancer: Collaborating with the Enemy. Front. Immunol. 2019, 10, 1805. [CrossRef] [PubMed]

26. Schafer, A.I. Thrombocytosis. N. Engl. J. Med. 2004, 350, 1211-1219. [CrossRef] [PubMed]

27. Mizrak, S.; Kucuk, S. Platelet levels and neutrophil to lymphocyte ratio in thyroid nodules with and without cancer diagnosis. Medicine 2020, 9, 16-20. [CrossRef]

28. Jianyong, L.; Zhihui, L.; Rixiang, G.; Jingqiang, Z. Using a nomogram based on preoperative serum fibrinogen levels to predict recurrence of papillary thyroid carcinoma. BMC Cancer 2018, 18, 390. [CrossRef] [PubMed]

29. Ozmen, H.K.; Askin, S.; Simsek, E.; Carlioglu, A.; Arikan, Ș.; Utlu, M. Mean Platelet Volume and Red Cell Distribution Width in Differentiated Thyroid Cancer Patients. Open Med. J. 2019, 6, 94-100. [CrossRef]

30. Yu, Y.J.; Li, N.; Yun, Z.Y.; Niu, Y.; Xu, J.J.; Liu, Z.P.; Liu, T.; Wang, R.T.; Yu, K.J. Preoperative mean platelet volume and platelet distribution associated with thyroid cancer. Neoplasma 2017, 64, 594-598. [CrossRef] [PubMed]

31. Templeton, A.J.; McNamara, M.G.; Šeruga, B.; Vera-Badillo, F.E.; Aneja, P.; Ocaña, A.; Leibowitz-Amit, R.; Sonpavde, G.; Knox, J.K.; Tran, B.; et al. Prognostic role of neutrophil-to-lymphocyte ratio in solid tumors: A systematic review and meta-analysis. J. Natl. Cancer Inst. 2014, 106, dju124. [CrossRef] [PubMed]

32. Kocer, D.; Karakukcu, C.; Karaman, H.; Gokay, F.; Bayram, F. May the Neutrophil/Lymphocyte Ratio Be a Predictor in the Differentiation of Different Thyroid Disorders? Asian Pac. J. Cancer Prev. 2015, 16, 3875-3879. [CrossRef] [PubMed]

33. Ceylan, Y.; Kumanlığlu, K.; Oral, A.; Ertan, Y.; Özcan, Z. The Correlation of Clinicopathological Findings and Neutrophil-toLymphocyte and Platelet-to-Lymphocyte Ratios in Papillary Thyroid Carcinoma. Mol. Imaging Radionucl. Ther. 2019, 28, 15-20. [CrossRef] [PubMed]

34. Chae, I.H.; Kim, E.K.; Moon, H.J.; Yoon, J.H.; Park, V.Y.; Lee, H.S.; Moon, J.; Kwak, J.Y. Preoperative High Neutrophil-Lymphocyte Ratio May Be Associated with Lateral Lymph Node Metastasis in Patients with Papillary Thyroid Cancers. Int. J. Thyroid. 2018, 11, 41-48. [CrossRef]

35. Feng, J.; Wang, Y.; Shan, G.; Gao, L. Clinical and prognostic value of neutrophil-lymphocyte ratio for patients with thyroid cancer: A meta-analysis. Medicine 2020, 99, e19686. [CrossRef] [PubMed]

36. Kim, S.M.; Kim, E.H.; Kim, B.H.; Kim, J.H.; Park, S.B.; Nam, Y.J.; Ahn, K.H.; Oh, M.Y.; Kim, W.J.; Jeon, Y.K.; et al. Association of the Preoperative Neutrophil-to-Lymphocyte Count Ratio and Platelet-to-Lymphocyte Count Ratio with Clinicopathological Characteristics in Patients with Papillary Thyroid Cancer. Endocrinol. Metab. 2015, 30, 494-501. [CrossRef] [PubMed]

37. Wakatsuki, K.; Matsumoto, S.; Migita, K.; Ito, M.; Kunishige, T.; Nakade, H.; Nakatani, M.; Kitano, M.; Sho, M. Preoperative Plasma Fibrinogen is Associated with Lymph Node Metastasis and Predicts Prognosis in Resectable Esophageal Cancer. World J. Surg. 2017, 41, 2068-2077. [CrossRef] [PubMed]

38. Palaj, J.; Kečkéš, Š.; Marek, V.; Dyttert, D.; Waczulíková, I.; Durdík, Š. Fibrinogen Levels Are Associated with Lymph Node Involvement and Overall Survival in Gastric Cancer Patients. Anticancer Res. 2018, 38, 1097-1104. [CrossRef] [PubMed]

39. Resende de Paiva, C.; Grønhøj, C.; Feldt-Rasmussen, U.; von Buchwald, C. Association between Hashimoto's Thyroiditis and Thyroid Cancer in 64,628 Patients. Front. Oncol. 2017, 7, 53. [CrossRef] [PubMed]

40. Korniluk, A.; Koper-Lenkiewicz, O.M.; Kamińska, J.; Kemona, H.; Dymicka-Piekarska, V. Mean Platelet Volume (MPV): New Perspectives for an Old Marker in the Course and Prognosis of Inflammatory Conditions. Mediat. Inflamm. 2019, $2019,9213074$. [CrossRef] [PubMed]

41. Pujani, M.; Chauhan, V.; Singh, K.; Rastogi, S.; Agarwal, C.; Gera, K. The effect and correlation of smoking with platelet indices, neutrophil lymphocyte ratio and platelet lymphocyte ratio. Hematol. Transfus. Cell Ther. 2020, in press. [CrossRef] [PubMed]

42. Shilpi, K.; Potekar, R.M. A Study of Platelet Indices in Type 2 Diabetes Mellitus Patients. Indian J. Hematol. Blood Transfus. 2018, 34, 115-120. [CrossRef] [PubMed] 